\title{
Antibody-mediated lung transplant rejection
}

\author{
Ramsey Hachem
}

Published online: 27 June 2012

(C) Springer Science+Business Media, LLC 2012

\begin{abstract}
Antibody-mediated rejection after lung transplantation remains enigmatic. However, emerging evidence over the past several years suggests that humoral immunity plays an important role in allograft rejection. Indeed, the development of donor-specific antibodies after transplantation has been identified as an independent risk factor for acute cellular rejection and bronchiolitis obliterans syndrome. Furthermore, cases of acute antibody-mediated rejection resulting in severe allograft dysfunction have been reported, and these demonstrate that antibodies can directly injure the allograft. However, the incidence and toll of antibodymediated rejection are unknown because there is no widely accepted definition and some cases may be unrecognized. Clearly, humoral immunity has become an important area for research and clinical investigation.
\end{abstract}

Keywords Antibody-mediated rejection $\cdot$ Lung transplantation $\cdot$ Hyperacute rejection $\cdot$ Bronchiolitis obliterans syndrome $\cdot$ Antibodies to human leukocyte antigens · Donor-specific antibodies · Complement component $4 \mathrm{~d}$ deposition

\section{Introduction}

Lung transplantation has become a definitive treatment option for patients with end-stage lung disease. Although the field is still relatively young, the number of transplants performed annually has steadily increased, and over 3,000

\section{R. Hachem $(\bowtie)$}

Division of Pulmonary \& Critical Care Medicine,

Washington University School of Medicine,

660 S. Euclid Ave., Campus Box 8052,

St. Louis, MO 63110, USA

e-mail: rhachem@dom.wustl.edu procedures were reported to the International Society for Heart and Lung Transplantation (ISHLT) Registry in 2009 [1]. Furthermore, outcomes have improved over time. In fact, the 5-year survival in the most recent era between 2000 and 2009 was $54 \%$, and this was significantly better than the 5-year survival in the previous era between 1995 and 1999 , which was $48 \%$, and the earliest era between 1988 and 1994 when the 5-year survival was also $48 \%$ [1]. Nonetheless, survival after lung transplantation remains significantly worse than after other solid organ transplants. Indeed, the 5-year survival after deceased-donor kidney transplantation was $85 \%$ in the latest Scientific Registry of Transplant Recipients (SRTR) Annual Report, and the 5year survival after heart transplantation was $75 \%$ [2]. In contrast, the 5-year survival after lung transplantation was $54 \%$ during the same time period in the SRTR Annual Report [2].

In the first year after lung transplantation, allograft failure and infections are the leading causes of death [1]. Beyond the first year, allograft failure due to bronchiolitis obliterans syndrome (BOS) and other forms of rejection is the leading cause of death accounting for approximately $50 \%$ of deaths [1]. Clearly, chronic allograft rejection is the primary obstacle to better long-term outcomes after lung transplantation. In general, rejection has been attributed primarily to cellular immunity, and conventional immunosuppression targeting T-cell proliferation and function has made transplantation clinically feasible. However, emerging data suggest that humoral immunity may play an important role in allograft rejection. Indeed, antibody-mediated rejection (AMR) is recognized as a form of rejection after kidney and heart transplantation [3-5]. Nonetheless, AMR after lung transplantation remains enigmatic, and there was no consensus on the diagnostic features of AMR in the latest ISHLT classification system of lung rejection [6]. The National 
Conference to Assess Antibody-Mediated Rejection in Solid Organ Transplantation proposed a paradigm of antibody responses after transplantation, and a definition of AMR based on the presence of donor-specific antibodies (DSA), complement component $4 \mathrm{~d}(\mathrm{C} 4 \mathrm{~d})$ deposition, tissue pathology, and clinical allograft dysfunction [7]. Multiple cases and case series of a unique form of allograft dysfunction that fulfill these criteria have been reported suggesting that AMR after lung transplantation may be an under-recognized cause

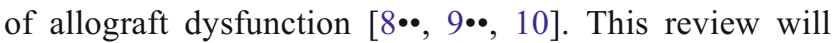
describe clinicopathological features of acute AMR and highlight different syndromes of antibody responses after lung transplantation.

\section{Hyperacute rejection}

Hyperacute rejection is caused by pre-existing DSA and occurs within minutes or hours of transplantation. It manifests clinically as fulminant allograft dysfunction with severe gas-exchange impairment, hemorrhagic pulmonary edema, and diffuse radiographic infiltrates. The pathogenesis involves the binding of pre-formed DSA to human leukocyte antigens (HLA) on donor endothelial cells and the subsequent activation of the classical complement cascade leading to the formation of the membrane attack complex and endothelial cell injury. This exposes the basement membrane and results in activation of the coagulation cascade leading to thrombosis and infarction. In addition, complement components $3 \mathrm{a}$ and $5 \mathrm{a}(\mathrm{C} 3 \mathrm{a}$ and $\mathrm{C} 5 \mathrm{a})$ are potent chemokines that recruit neutrophils and other inflammatory cells that further augment the allograft injury. Hyperacute rejection has become rare because screening methodologies for HLA antibodies before transplantation have become more sensitive and specific preventing transplantation across an HLA barrier. Nonetheless, it is a widely accepted form of rejection after lung transplantation and demonstrates that antibodies can directly injure the lung allograft. The pathology is characterized by severe acute lung injury with neutrophilic infiltration of the alveolar septae, fibrinoid necrosis, and hemorrhagic infarction [11-14]. In a case report of hyperacute rejection, Choi and colleagues demonstrated antibody deposition on the endothelial surface and within vessel walls [11]. Similarly, Frost and colleagues illustrated endothelial cell damage and neutrophils attached to endothelial cells of alveolar septal capillaries with electron microscopy in another case of hyperacute rejection [12].

Despite intensive treatment with various combinations of intravenous immune globulin (IVIG), plasmapheresis, cyclophosphamide, rituximab, and anti-thymocyte globulin, outcomes of hyperacute rejection have been poor and allograft loss is common. However, Bittner and colleagues reported a successful result using plasmapheresis and anti- thymocyte globulin in a case report of hyperacute rejection [13]. Fortunately, prevention of hyperacute rejection with more sensitive HLA antibody detection techniques has made this form of rejection rare.

\section{De novo HLA antibodies}

Multiple studies have identified the development of HLA antibodies after transplantation as a significant predictor of poor outcomes. The de novo development of HLA antibodies has been identified as a significant risk factor for BOS, independent of other risk factors, and the development of antibodies precedes the onset of BOS $[15,16]$. Furthermore, HLA antibodies have been associated with recurrent and high-grade acute (cellular) rejection and lymphocytic bronchiolitis $[17,18]$. These findings underscore the potential role for humoral immunity in allograft rejection, but an alternative paradigm may be that antibodies are merely an epiphenomenon of cellular immunity or a biomarker for rejection. However, several studies support a pathogenic role for HLA antibodies. First, an in vitro study demonstrated that class I HLA antibodies can activate airway epithelial cells and induce the release of soluble growth factors, stimulate fibroblast proliferation, and induce epithelial cell apoptosis, all central events in the pathogenesis of obliterative bronchiolitis [19]. Furthermore, in a murine study, tracheal allografts were transplanted into Rag 1-deficient mice (lacking functional $\mathrm{T}$ - and B-cells) and the mice were treated with HLA antibodies [20]. The allografts developed epithelial cell damage followed by denudation and luminal obliteration and fibrosis [20]. In addition, this resulted in macrophage and neutrophil chemotaxis into the allograft. In another murine study, HLA antibodies were administered in the airways of native lungs, and this resulted in cellular infiltration, epithelial hyperplasia, and fibroproliferation resulting in airway luminal narrowing [21]. Collectively, these findings suggest that HLA antibodies have a pathogenic role in BOS development and are not merely an epiphenomenon of cellular immunity.

Based on these clinical and experimental data, we hypothesized that early elimination or inactivation of DSA would mitigate the risk of BOS and developed a protocol to screen for DSA after transplantation and a preemptive treatment regimen consisting of rituximab and/or IVIG [22••]. Surprisingly, the incidence of de novo DSA after transplantation was $56 \%$, and the majority of patients who developed DSA did so in the first 90 days after transplantation [22••]. Those who developed DSA were treated with rituximab and/or IVIG before the onset of allograft dysfunction, and approximately $60 \%$ cleared the DSA [22••]. Importantly, those who cleared the DSA were significantly less likely to develop BOS and had better survival than 
those who had persistent DSA [22••]. These findings suggest that DSA depletion ameliorates the risk of BOS. However, this was not a randomized controlled trial and the treatment group was not compared to an untreated group of patients who developed DSA. Furthermore, it is unknown if DSA may clear over time with maintenance immunosuppression. Therefore, it is difficult to conclude that DSA depletion was a treatment effect, and a randomized controlled trial is needed to critically evaluate the role of this strategy. Nevertheless, there is considerable evidence that suggests that HLA antibodies have a pathogenic role in BOS development.

\section{Acute antibody-mediated rejection}

Acute AMR after lung transplantation remains enigmatic and there is no consensus on the characteristic clinicopathologic features. However, several reports describing a unique form of allograft rejection that is consistent with the definition of AMR proposed by The National Conference to Assess Antibody-Mediated Rejection in Solid Organ Transplantation have been published. In the earliest series, Badesch and colleagues described 5 patients who developed pulmonary capillaritis after transplantation [23]. All presented with acute allograft dysfunction and diffuse alveolar hemorrhage. There was a transient response to more intensive immunosuppression and plasmapheresis, but 2 of the 5 patients died of allograft failure [23]. The clinicopathological findings were not correlated with HLA antibody testing, but it is likely that these patients had acute AMR given the similarities with subsequently reported cases. Magro and colleagues described the histologic features of 22 patients who developed a similar capillaritis syndrome [24]. The pathology was remarkable for septal capillary necrosis with deposition of complement components, including $\mathrm{C} 4 \mathrm{~d}$, and immunoglobulin G. However, none of the recipients had detectable HLA antibodies although older and less sensitive detection methods were used. Nonetheless, antibodies to endothelial cells were documented and there was clinical improvement with plasmapheresis [24]. Interestingly, the endothelial cell antigen specificity was not determined in this study and the target endothelial cells were not donor derived. This suggests that the antibodies may have been directed to self-antigens on endothelial cells and raises the suspicion of an autoimmune process.

Two recent case reports from independent centers describe similar clinicopathological features of acute AMR after lung transplantation $[8 \bullet \bullet, 9 \bullet \bullet]$. In the first case, an 8month old infant underwent bilateral lung transplantation for alveolar proteinosis and did well initially [8••]. The recipient was not allosensitized before transplantation and the direct crossmatch was negative, but he developed respiratory

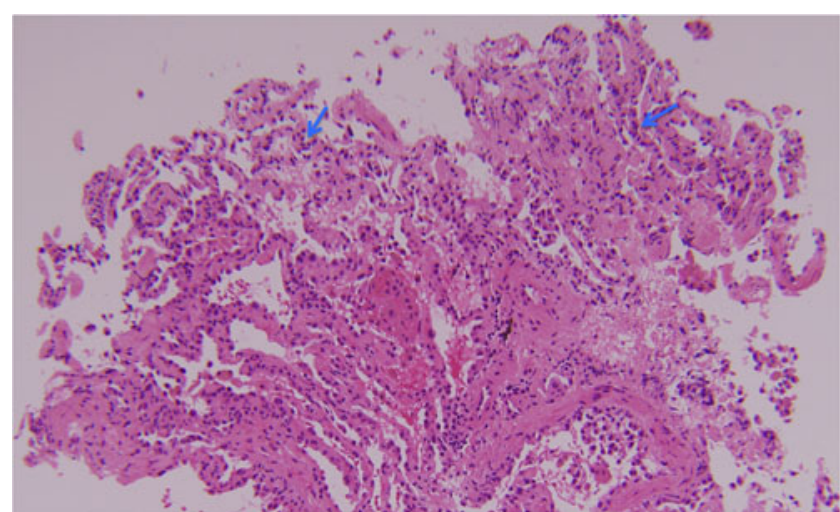

Fig. 1 This transbronchial lung biopsy from a patient with acute antibody-mediated rejection illustrates alveolar septal neutrophilia (blue arrows) suspicious for capillaritis

failure and diffuse pulmonary infiltrates 2 weeks after transplantation [8・•]. A surgical lung biopsy demonstrated neutrophils infiltrating alveolar septae and capillary walls consistent with capillaritis and immunofluorescence and immunohistochemistry showed diffuse $\mathrm{C} 4 \mathrm{~d}$ deposition in alveolar capillaries $[8 \cdot \bullet]$. In addition, the recipient was found to have de novo DSA by the Luminex assay [8••]. There was no response to high-dose steroids and basiliximab, but the recipient responded well to rituximab with clearance of the DSA and resolution of the allograft dysfunction $[8 \cdot \bullet$. We reported similar findings in a case of acute AMR in an adult recipient 1 month after transplantation $[9 \bullet \bullet$. Again, the recipient was not allosensitized before transplantation and the direct crossmatch was negative at the time of transplantation. The initial post-operative course was uncomplicated, but the recipient developed acute respiratory failure; transbronchial lung biopsy showed acute lung injury with neutrophil infiltration of the alveolar septae with capillaritis and $\mathrm{C} 4 \mathrm{~d}$ deposition in the alveolar capillaries by immunohistochemistry [9*•]. The recipient was also found to have de novo DSA by the Luminex assay [9••]. Treatment

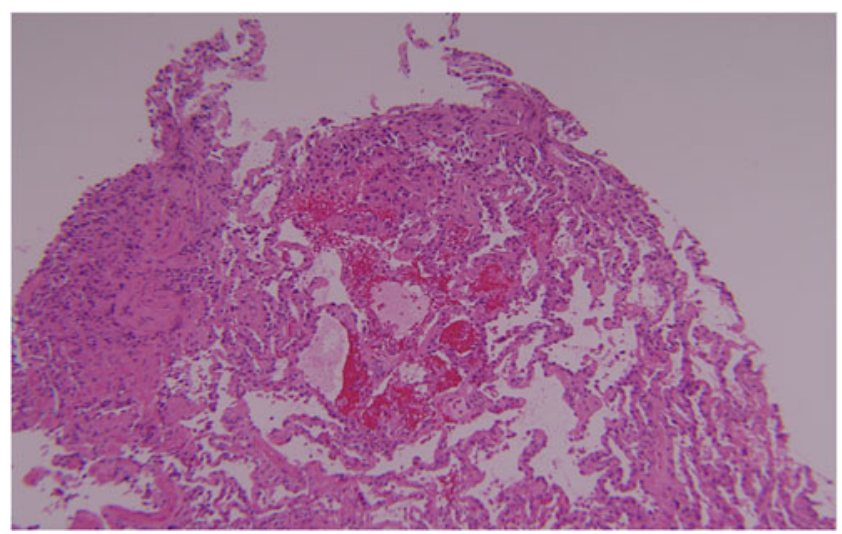

Fig. 2 Another transbronchial lung biopsy from the same patient with acute antibody-mediated rejection demonstrates hemorrhage and focal fibrin deposition 
was multifaceted consisting of IVIG, plasmapheresis, and rituximab, and the recipient responded well clinically al-

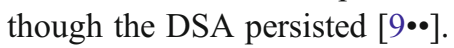

We subsequently reported our experience with acute AMR in a cohort of 16 patients [10]. Five were allosensitizied before transplantation, but the reactive HLA were avoided in their respective donors and the direct crossmatch was negative at the time of transplantation in all 16 recipients [10]. Patients presented with hypoxemia and pulmonary infiltrates, and 9 required mechanical ventilation. Overall, the time of onset was early, occurring a mean 283 days after transplantation, and 11 developed AMR within the first year. All recipients had de novo DSA. The predominant pathology was acute lung injury with diffuse alveolar damage, and features of capillaritis were identified in 8 cases [10]. Figure 1 illustrates an example of alveolar septal neutrophilia and Fig. 2 demonstrates hemorrhage and focal fibrin deposition. All patients had C4d deposition by immunohistochemistry in the alveolar capillary endothelium demonstrating complement activation. Patients were treated with rituximab and IVIG, and 5 patients were also treated with plasmapheresis. Thirteen patients improved and 3 died of refractory AMR, but 8 of the 13 survivors developed BOS within 1 year of the diagnosis of AMR [10]. Based on these findings, we proposed that the following features are characteristic of acute AMR: circulating DSA, acute lung injury with or without capillaritis, $\mathrm{C} 4 \mathrm{~d}$ deposition in the alveolar capillaries, and overt allograft dysfunction [10]. However, this definition needs to be validated in a multicenter fashion. Furthermore, the pathology of acute lung injury is nonspecific, and this is especially true when capillaritis is not identified. Nonetheless, capillaritis emerged as an important feature of AMR based on the histology of hyperacute rejection, but it is not clear that capillaritis is the sine qua non of pulmonary AMR. Indeed, capillaritis is not seen in some cases that fulfill the other diagnostic criteria. In such cases, it is possible that the capillary endothelium is the initial target of antibody-mediated injury, but the morphologic features of capillaritis become obscured by the acute lung injury as the rejection progresses. In addition, the inter-reader and intrareader reliability of capillaritis and the sensitivity of transbronchial lung biopsy are unknown. Similarly, the interpretation of C4d deposition in the lung is fraught with complications.

Recently, Yousem and colleagues conducted a retrospective study to identify morphologic features associated with DSA in the setting of allograft dysfunction [25]. Seventeen of the 23 patients with DSA had acute cellular rejection (2 had grade A2, 13 had grade A3, and 1 had grade A4), and 5 of the 23 had acute and organizing lung injury [25]. Importantly, capillaritis was seen in a minority of cases and was associated with cellular rejection. In addition, the authors note that in a separate cohort of 7 transplant recipients with capillaritis, none had DSA [25]. This suggests that capillaritis may not be specific for AMR. Similarly, C4d deposition was neither sensitive nor specific; 13 of the 17 patients with acute cellular rejection and DSA had C4d deposition, and 6 of 26 patients with acute cellular rejection but no DSA had C4d deposition [25]. However, it should be noted that this study was not designed to identify histologic criteria for AMR but to identify morphologic features associated with circulating DSA.

Collectively, these studies suggest that no morphologic findings are specific for AMR, and the diagnosis is best made using serologic, clinical, and histologic data together. This is distinct from the diagnosis of acute cellular rejection and lymphocytic bronchiolitis, which is based solely on histology.

\section{Conclusions}

There is compelling evidence that antibodies can directly injure the lung allograft. Hyperacute rejection is fulminant and often results in allograft failure and loss. The development of de novo DSA after transplantation also portends a poor prognosis with an increased risk of high-grade and refractory acute cellular rejection, lymphocytic bronchiolitis, and BOS. Furthermore, de novo DSA cause acute AMR in a small minority of patients. The mechanisms that determine the effect of DSA on the allograft are currently unknown. It is possible that DSA associated with an increased risk of BOS do not activate complement. Instead, these antibodies may activate epithelial cells resulting in the release of fibrogenic growth factors that mediate the development of BOS $[19,20]$. On the other hand, complementactivating DSA would cause acute AMR or hyperacute rejection. However, it is not clear that the different effects are solely based on the ability of DSA to activate complement and other unknown factors may contribute to the ultimate result. Clearly, the role of humoral immunity in lung allograft rejection requires additional study, and a multidisciplinary and multi-center approach is necessary.

Disclosure No potential conflicts of interest relevant to this article were reported.

\section{References}

Papers of particular interest, published recently, have been highlighted as:

•• Of major importance

1. Christie JD, Edwards LB, Kucheryavaya AY, et al. The registry of the International Society for Heart and Lung Transplantation: twenty-eighth adult lung and heart-lung transplant report-2011. J Heart Lung Transplant. 2011;30:1104-22. 
2. Organ Procurement and Transplantation Network (OPTN) and Scientific Registry of Transplant Recipients (SRTR). OPTN/ SRTR 2010 Annual Data Report. Rockville, MD: Department of Health and Human Services, Health Resources and Services Administration, Healthcare Systems Bureau, Division of Transplantation; 2011.

3. Solez K, Colvin RB, Racusen LC, et al. Banff 07 classification of renal allograft pathology: updates and future directions. Am J Transplant. 2008;8:753-60.

4. Sis B, Mengel M, Haas M, et al. Banff 09 meeting report: antibody mediated graft deterioration and implementation of Banff working groups. Am J Transplant. 2010;10:464-71.

5. Berry GJ, Angelini A, Burke MM, et al. The ISHLT working formulation for pathologic diagnosis of antibody-mediated rejection in heart transplantation: evolution and current status (20052011). J Heart Lung Transplant. 2011;30:601-11.

6. Stewart S, Fishbein MC, Snell GI, et al. Revision of the 1996 working formulation for the standardization of nomenclature in the diagnosis of lung rejection. J Heart Lung Transplant. 2007;26:1229-42.

7. Takemoto SK, Zeevi A, Feng S, et al. National conference to assess antibody-mediated rejection in solid organ transplantation. Am J Transplant. 2004;4:1033-41.

8. •- Astor TL, Galantowicz M, Phillips A, Palafox J, Baker P. Pulmonary capillaritis as a manifestation of acute humoral allograft rejection following infant lung transplantation. Am J Transplant. 2009;9:409-12. This manuscript is a thorough case report of acute antibody-mediated rejection after lung transplantation in an infant.

9. •- Morrell MR, Patterson GA, Trulock EP, Hachem RR. Acute antibody-mediated rejection after lung transplantation. J Heart Lung Transplant. 2009;28:96-100. This manuscript reports similar findings of acute antibody-mediated rejection after lung transplantation in an adult.

10. Hachem RR, Ritter JP, Yusen RD, et al. Antibody-mediated rejection after lung transplantation [Abstract]. J Heart Lung Transplant. 2011;30:S36-7. Presented at The International Society for Heart and Lung Transplantation $31^{\text {st }}$ Annual Meeting and Scientific Sessions. San Diego, CA; April 13-16, 2011.

11. Choi JK, Kearns J, Palevsky HI, et al. Hyperactue rejection of a pulmonary allograft. Am J Respir Crit Care Med. 1999;160:1015-8.

12. Frost AE, Jammal CT, Cagle PT. Hyperacute rejection following lung transplantation. Chest. 1996;110:559-62.

13. Bittner HB, Dunitz J, Hertz M, Bolman MR, Park SJ. Hyperacute rejection in single lung transplantation-case report of successful management by means of plasmapheresis and antithymocyte globulin treatment. Transplantation. 2001;71:649-51.
14. Masson E, Stern M, Chabod J, et al. Hyperacute rejection after lung transplantation caused by undetected low-titer anti-HLA antibodies. J Heart Lung Transplant. 2007;26:642-5.

15. Sundaresan S, Mohanakumar T, Smith MA, et al. HLA-A locus mismatches and development of antibodies to HLA after lung transplantation correlate with the development of bronchiolitis obliterans syndrome. Transplantation. 1998;65:648-53.

16. Jaramillo A, Smith MA, Phelan D, et al. Development of ELISAdetected anti-HLA antibodies precedes the development of bronchiolitis obliterans syndrome and correlates with progressive decline in pulmonary function after lung transplantation. Transplantation. 1999;67:1155-61.

17. Girnita AL, McCurry KR, Iacono AT, et al. HLA-specific antibodies are associated with high-grade and persistentrecurrent lung allograft acute rejection. J Heart Lung Transplant. 2004;23:1135-41.

18. Girnita AL, Duquesnoy R, Yousem SA, et al. HLA-specific antibodies are risk factors for lymphocytic bronchiolitis and chronic lung allograft dysfunction. Am J Transplant. 2005;5:131-8.

19. Jaramillo A, Smith CR, Maruyama T, Zhang L, Patterson GA, Mohanakumar T. Anti-HLA class I antibody binding to airway epithelial cells induces production of fibrogenic growth factors and apoptotic cell death: a possible mechanism for bronchiolitis obliterans syndrome. Hum Immunol. 2003;64:521-9.

20. Maruyama T, Jaramillo A, Narayanan K, Higuchi T, Mohanakumar T. Induction of obliterative airway disease by anti-HLA class I antibodies. Am J Transplant. 2005;5:2126-34.

21. Fukami N, Ramachandran S, Saini D, et al. Antibodies to MHC class I induce autoimmunity: role in the pathogenesis of chronic rejection. J Immunol. 2009;182:309-18.

22. •• Hachem RR, Yusen RD, Meyers BF, et al. Anti-human leukocyte antigen antibodies and preemptive antibody-directed therapy after lung transplantation. J Heart Lung Transplant. 2010;29:97380. This study suggests that preemptive depletion of HLA antibodies may ameliorate the risk of bronchiolitis obliterans syndrome.

23. Badesch DB, Zamora M, Fullerton D, et al. Pulmonary capillaritis: a possible histologic form of acute pulmonary allograft rejection. J Heart Lung Transplant. 1998;17:415-22.

24. Magro CM, Deng A, Pope-Harman A, et al. Humorally mediated posttransplantation septal capillary injury syndrome as a common form of pulmonary allograft rejection: a hypothesis. Transplantation. 2002;74:1273-80.

25. Yousem SA, Zeevi A. The histology of lung allograft dysfunction associated with the development of donor-specific HLA antibodies. Am J Surg Pathol. 2012, April 16 [Epub ahead of print]. 